\title{
A new set of analytical formulae for the computation of the bootstrap current and the neoclassical conductivity in tokamaks
}

\author{
A. Redl, ${ }^{1,2}$, a) C. Angioni, ${ }^{1}$ E. Belli, ${ }^{3}$ O. Sauter, ${ }^{4}$ the ASDEX Upgrade Team, ${ }^{\text {b) }}$ and the EUROfusion MST1 \\ Team ${ }^{\mathrm{c})}$ \\ ${ }^{1)}$ Max-Planck-Institute for Plasma Physics, 85748 Garching, Germany \\ 2) Ludwig Maximilian University of Munich, 80539 Munich, Germany \\ 3) General Atomics, P.O. Box 85608, San Diego, CA 92186-5608, USA \\ 4) École Polytechnique Fédérale de Lausanne, 1015 Lausanne, Switzerland
}

(Dated: 13 February 2021)

A new set of analytical formulae for calculating the bootstrap current and the neoclassical conductivity in tokamak experiments is presented. Previous works comparing the widely used Sauter model with results of recently developed numerical neoclassical solvers have shown the Sauter model to be inaccurate at higher collisionalities typical of tokamak edge pedestals and in the presence of impurities. Thus, its applicability, particularly for the analysis of the highly interesting as well as highly complex plasma edge, is limited. For a revised set of analytical formulae, the procedure to determine the analytic formulae described by Sauter is repeated with the more accurate and more reliable numerical code NEO[E. Belli, Plasma Physics and Controlled Fusion 54, 015015 (2012)]. For the determination of the respective bootstrap current coefficient, the linearity of neoclassical transport is exploited. This new set of analytical formulae consists of the same analytical structure as the original set of analytical formulae published in Sauter[O. Sauter, Physics of Plasma 6, 2834 (1999); O. Sauter, Physics of Plasma 9, 5140 (2002)] and also continues to use only three neoclassical key parameters: the fraction of trapped particles $f_{\text {trap }}$, the collisionality $v_{\sigma}^{*}$ and the effective charge number $Z_{\text {eff }}$.

\section{INTRODUCTION}

The bootstrap current, a neoclassical effect, is expected to play a crucial role in future fusion power plants[1,2]. This parallel electric current, generated by the plasma itself in the presence of radial pressure gradients, can provide a significant current contribution in advanced or steady-state scenarios[3]. Numerical codes for computing neoclassical transport by solving the drift-kinetic Fokker-Planck equation have been developed starting from the late 80s. However, in the framework of integrated modelling, even recently developed numerical neoclassical transport codes are not very practical, as they can still require a large amount of computing power and computing time. For fast applications on large numbers of cases, it is more convenient to adopt analytical models, which use a few suitable key parameters in order to compute accurately and realiably the most important neoclassical transport contributions. The Sauter model[4,5] is a widely used set of analytic formulae for the computation of the bootstrap current and the neoclassical conductivity. These analytical formulae were obtained by best fitting the results of the codes CQL3D[6,7] and CQLP[8] for arbitrary collisionality and magnetic equilibria. For its application, the Sauter model needs three neoclassical key input parameters, the fraction of trapped particles $f_{\text {trap }}[9]$, the collisionality $v_{\sigma}^{*}[10]$ and the effective charge number $Z_{\text {eff }}[10]$. More recently, additional analytical models have been developed based on the Sauter model[11,12]. These more recent models introduce further fit parameters of additional physical effects in order to improve the accuracy of the original Sauter model.

\footnotetext{
a)Electronic mail: Andreas.Redl@ipp.mpg.de

b) See author list of H. Meyer et al. 2019 Nuclear Fusion 59112014

${ }^{\text {c) }}$ See author list of B. Labit et al. 2019 Nuclear Fusion 59086020
}

The discrepancy between accurate Fokker-Planck neoclassical numerical results and the analytical Sauter model, for higher collisionality, and particularly in the pedestal, was first identified simultaneously in Koh[11] and in Landreman[13] and later in Belli[14]. In particular, Refs.[11,13] show a nearly identical result for a DIII-D like pedestal case, demonstrating that the Sauter model overestimates the bootstrap current in the edge pedestal region. In Belli[14], the limitations of two analytical models, the Sauter model and the KCK12 model[11], a recently developed set of analytical formulae based on the Sauter model, are compared with the recently developed numerical code NEO $[15,16,17]$. This study shows that for a 2-species plasma at low collisionality the Sauter model is still very accurate, but, consistently with previous works[11,13], this study also confirms that at high collisionality the bootstrap current is significantly overestimated. This limits the applicability of the Sauter model at the plasma edge in particular. Additionally, the multi-species description of the Sauter model for three or more species is found to be quite incorrect. These realizations motivate a revision of the Sauter model by adopting the same fitting procedure, which was originally used, but considering the numerical results of the more recent NEO code as reference. For this reason, the resulting revised formulae proposed here are still based on the general orderings of conventional neoclassical transport theory[10]. It is known that for the ions these orderings are not always satisfied in the tokamak edge pedestal region and further corrections may be required, which are not considered here[11,12,13,14].

In Section II the drift-kinetic solver NEO and its numerical approach are briefly discussed. In the subsequent section III, the exploited approach is discussed to determine the bootstrap current coefficient from the NEO results. The profiles from NEO and the ones of the analytical models are presented and compared. Finally, the newly obtained analytic formulae of 
the bootstrap current and neoclassical conductivity are presented. In Section IV the original Sauter model and the revised model are applied to a typical plasma discharge of ASDEX Upgrade in order to compare the predictions with NEO. Section V contains the summary and an outlook.

\section{THE NEOCLASSICAL SOLVER NEO}

\section{A. The NEO numerical approach}

The code NEO is a multi-species drift-kinetic solver, which solves the first-order drift-kinetic-Poisson equations. NEO solves the full-linearized Fokker-Planck collision operator without any approximations beyond the drift-ordering parameter $\left(\rho_{*}=\rho_{\mathrm{i}} / a \ll 1\right)$, where $\rho_{\mathrm{i}}=\left(m_{\mathrm{i}} v_{\perp, \mathrm{i}}\right) /\left(q_{\mathrm{i}} B\right)$ is the Larmor radius[18] and $a$ the plasma minor radius. NEO computes collision dynamics, where complete cross-species collisional coupling for arbitrary mass ratio and arbitrary number of species are considered. Additionally, general flux-surface shape, including up-down asymmetry, is handled. A special feature is the possibility to retain the full sonic rotation and centrifugal terms. These effects are not considered in this particular analysis.

For solving the drift-kinetic-Poisson equations, the code NEO uses a novel numerical algorithm, which consists of a mixture of spectral methods and finite-difference schemes. The distribution function of first-order is expanded using a Legendre polynomial basis in the cosine of the pitch angle $\xi=$ $v_{\|} / v$ and a generalized Laguerre polynomial with basis $x$. $x=v /\left(\sqrt{2} v_{\mathrm{Th}, \sigma}\right)$ is the relative kinetic energy and introduced as a parameter in the NEO routine. Therefore, according to Ref.[17], the distribution function is described as:

$$
f_{1, \sigma}=f_{0, \sigma} \sum_{\mathrm{l}=0}^{\mathrm{N}_{\xi}} \sum_{\mathrm{m}=0}^{\mathrm{N}_{\mathrm{x}}} \widehat{f}_{1, \sigma}^{\mathrm{m}} P_{1}(\xi) L_{\mathrm{m}}^{\mathrm{k}(\mathrm{l})+1 / 2}\left(x_{\sigma}^{2}\right) x_{\sigma}^{\mathrm{k}(\mathrm{l})}
$$

where a suitable choice for $\mathrm{k}(\mathrm{l})$ must be made and $\mathrm{N}_{\xi}$ and $\mathrm{N}_{x}$ denote the number of polynomials for the respective expansion basis in $\xi$ and $x_{\sigma} . \sigma$ denotes the considered ion species. A detailed description of the choice of $\mathrm{k}(\mathrm{l})$ and the approach is given in Ref.[17]. This approach allows one to reduce the problem solving the Fokker-Planck equation to a $\mathrm{NxN}$ matrix equation, where $N$ is defined as $N=N_{\text {species }} N_{\theta} N_{\xi} N_{x}$. $\mathrm{N}_{\text {species }}$ denotes the number of involved species, $\mathrm{N}_{\theta}$ the number of gridpoints in the $\theta$-grid range, $N_{\xi}$ the number of $\xi$ polynomials in the computational domain and $\mathrm{N}_{\mathrm{x}}$ the number of energy-polynomials in the computational domain. As one can see the size of the solving matrix depends strongly on the parameters of $\mathrm{N}_{\xi}$ and $\mathrm{N}_{\mathrm{x}}$. Because of this connection, the dependence of the bootstrap current on the choice of the parameters $\mathrm{N}_{\xi}$ and $\mathrm{N}_{\mathrm{x}}$ is considered in the next subsection in detail.
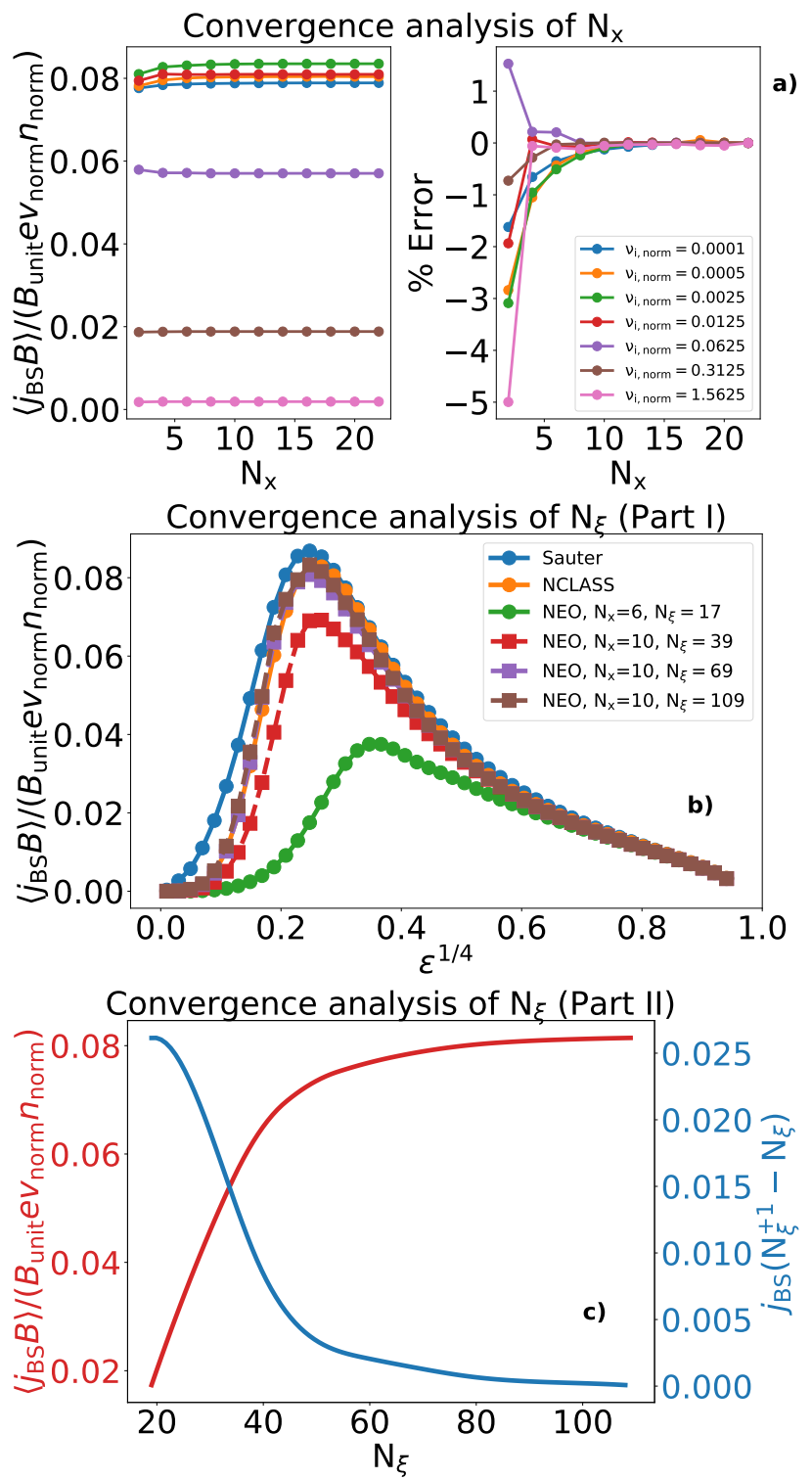

FIG. 1. Dependence of the normalized bootstrap current (a,left) and corresponding relative percentage deviation (a,right) as a function of the number of Laguerre polynomials $\mathrm{N}_{x}$ in the NEO velocity space discretization for different values of normalized collisionality $v_{\mathrm{i}, \text { norm }}=v_{\mathrm{i}} \cdot a / v_{\mathrm{th}, \mathrm{i}}$ as reported in the legend, and computed at $\varepsilon^{1 / 2}=0.3891$. The relative deviation in $\%$ is computed with respect to the result obtained with the largest value of $\mathrm{N}_{x}$. Profiles of the normalised bootstrap current (b) calculated with NEO for different values of $\mathrm{N}_{\xi}$ compared with the results of NCLASS[19] (orange line with circled markers) and the Sauter model (blue line with circled markers) are plotted against $\varepsilon^{1 / 4}$. It is mentioned that the change of $\mathrm{N}_{\xi}$ and $\mathrm{N}_{\mathrm{x}}$ does not effect the results of NCLASS and the Sauter model. The convergence behaviour of the code NEO (c) for various values of $\mathrm{N}_{\xi}$ is presented. The physical input parameters are taken from Fig. 1 (b) at $\varepsilon^{1 / 4} \approx 0.2$. The red line denotes the bootstrap current for different values of $\mathrm{N}_{\xi}$, the blue one denotes the difference of the bootstrap current density of two neighboring values of $N_{\xi}$. 


\section{B. Impact of the numerical resolution in NEO on the computation of the bootstrap current}

NEO input files are prepared for 2-species calculations. Circular Miller geometry and constant logarithmic gradients are considered in input. The parameters $\mathrm{N}_{\xi}$ and $\mathrm{N}_{\mathrm{x}}$ are varied over a large range, starting from the respective default values (17 and 6) to much larger values, respectively. The results of these calculations are plotted in Fig. 1. In Fig. 1 (a), the normalised bootstrap current calculated with NEO at $\varepsilon^{1 / 2}=$ 0.3891 , fixed value of $\mathrm{N}_{\xi}=29$ and for different values of the normalised ion-ion collision frequency $v_{\mathrm{i}, \mathrm{norm}}=v_{\mathrm{i}} \cdot a / v_{\mathrm{Th}, \mathrm{i}}$ is plotted. The right plot in Fig. 1 (a) shows the percentage relative deviation of the bootstrap current for different values of $\mathrm{N}_{x}$ from the bootstrap current obtained with the highest value of $\mathrm{N}_{x}$. These plots show that, at least for the range of parameters that have been considered in this study, the convergence in number of generalized Laguerre polynomials is already reached at relatively small values. To obtain convergence in the low collisionality limit, which is the most demanding in terms of resolution to reach converged, well resolved results, only values $\approx 10$ are required for $\mathrm{N}_{\mathrm{x}}$. The choice of $\mathrm{N}_{x}$ negligibly affects the results of NEO for the computation of the bootstrap current. In all of the calculations, the value $\mathrm{N}_{x}=10$ has been used. In Fig. 1 (b), the ion-ion collision frequency is kept constant at a very low value, consistent with the banana collisional regime. Consequently, the collisionality $v_{\sigma}^{*}$ varies now only with $\varepsilon$ (according to Eqs. (18b) and (18c) in Ref.[4]), $\varepsilon=r / R_{0}$ denotes the inverse aspect ratio. According to this plot, it is easy to see that the choice of $\mathrm{N}_{\xi}$ is essential for a correct calculation of the bootstrap current with NEO. By taking the default value of $\mathrm{N}_{\xi}$ the NEO results (green line with circled markers) strongly underestimate the respective results of NCLASS and the Sauter model. With strongly increased values of $\mathrm{N}_{\xi}$ the NEO results converge into the range of the results of NCLASS and the Sauter model. We have verified that this requirement in very high resolution in $\mathrm{N}_{\xi}$ is particularly strong at low collisionality, whereas it becomes less stringent with increasing collisionality. This is why here we focus on the low collisionality limit, which is the most demanding.

This problem can be related to the Legendre polynomial expansion in $\xi$. The required number of $\xi$-polynomials increases with decreasing $\varepsilon$ and prevents the numerically recovery of the analytic results in the low collisionality regime with NEO. The particle distributions become discontinous at the trapped-passing boundary in the collisionless limit, while in the high collisional limit, this discontinuity is normally compensated and smoothed through collisions. In the collisionless limit there is no collision mechanism assuring the continuity of the distribution function between the trapped-passing boundary in velocity space. This means in the collisionless limit more Legendre polynomials are required to resolve the near-discontinuity. It is now important to examine which convergence value should be chosen for $\mathrm{N}_{\xi}$ in order to create reliable bootstrap current results. Fig. 1 (c) presents this analysis. The physical input parameters are taken at position $\varepsilon^{1 / 4} \approx 0.2$ from Fig. 1 (b). The red line denotes the bootstrap current for different values of $\mathrm{N}_{\xi}$, the blue one denotes the difference of
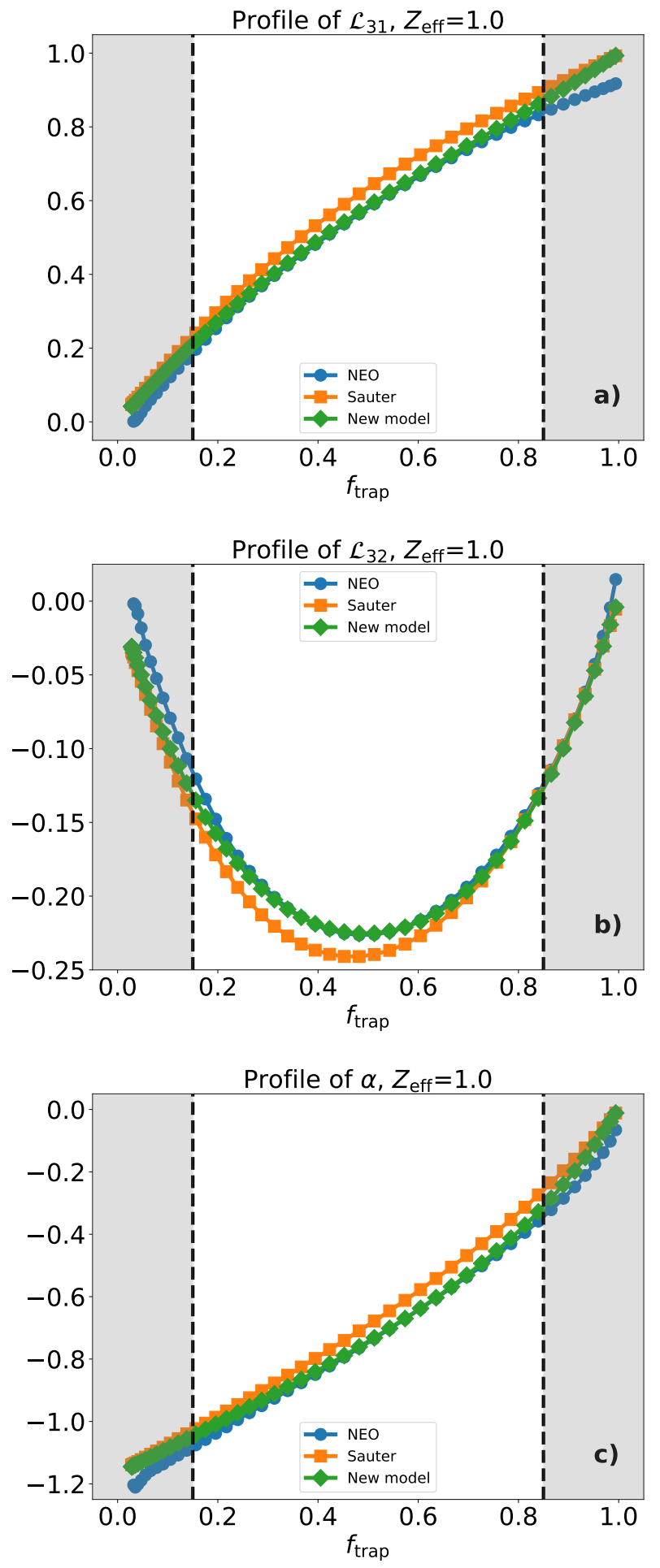

FIG. 2. Profiles of $\mathscr{L}_{31}$ (a), $\mathscr{L}_{32}$ (b) and $\alpha$ (c) in comparison with NEO (solid blue line with circled markers), the original Sauter model (solid orange line with squared markers) and the newly fitted model (solid green line with diamond markers) in the deep banana regime against $f_{\text {trap }}$ for constant $v_{\sigma}^{*}\left(v_{\mathrm{e}}^{*} \approx 4 \cdot 10^{-5}\right.$ and $\left.v_{\mathrm{i}}^{*} \approx 10^{-5}\right), Z_{\mathrm{eff}}=1.0$ and $\mathrm{N}_{\xi}=69$. It is noted that the grey highlighted areas represents the regions, where the analytic expressions exhibit structural disagreements from the NEO results, which cannot be overcome or avoided. 
the bootstrap current of two neighboring values of $\mathrm{N}_{\xi}$. According to the relation $j_{\mathrm{BS}}\left(\mathrm{N}_{\xi}^{+1}-\mathrm{N}_{\xi}\right) / j_{\mathrm{BS}}\left(\mathrm{N}_{\xi}\right)$, a required difference of $\approx 1 \%$ between two values of $\mathrm{N}_{\xi}$ is achieved around 75. For the large number of calculations performed in this work, the value $\mathrm{N}_{x} \approx 70$ has been used for 2 -species calculations. For 3 -species calculations, the value $\mathrm{N}_{x} \approx 40$ has been used.

\section{THE IMPROVEMENT OF THE ANALYTIC FORMULAE}

\section{A. Determination of the bootstrap current coefficients from the NEO outputs}

The linearity of neoclassical transport theory is exploited to derive the individual transport coefficients from the results of the code NEO by cleverly selecting the input parameters. For this purpose, Eq. (5) from Ref.[4] is expanded to show the individual gradients with their associated bootstrap current coefficients. Eq. (2) presents several general expression of the bootstrap current and the neoclassical conductivity:

$$
\begin{array}{r}
\left\langle j_{\|} B\right\rangle=\sigma_{\text {neo }}\left\langle E_{\|} B\right\rangle-I(\psi)\left(p \mathscr{L}_{31} \frac{\partial \ln n}{\partial \psi}+p_{\mathrm{e}}\left(\mathscr{L}_{31}+\mathscr{L}_{32}\right)\right. \\
\left.\frac{\partial \ln T_{\mathrm{e}}}{\partial \psi}+p_{\mathrm{i}}\left(\mathscr{L}_{31}+\mathscr{L}_{34} \alpha\right) \frac{\partial \ln T_{\mathrm{i}}}{\partial \psi}\right),
\end{array}
$$

where the left side of Eq. (2) denotes the flux surface averaged parallel electric current density, the first term on the right side denotes the neoclassical conductivity multiplied by the flux surface averaged inductive parallel electric field. The following terms denote the bootstrap current driven by the contributions of the pressure gradient of the involved species. In this presented approach the occurring density gradients of ions and electrons are set equal. From Eq. (2) one can now derive the profiles of the respective bootstrap current coefficients by selecting the related gradients as follows:

$$
\begin{gathered}
\left\langle j_{\|} B\right\rangle=-I(\psi) p \mathscr{L}_{31} \frac{\partial \ln n}{\partial \psi} \rightarrow \mathscr{L}_{31}=\frac{-\left\langle j_{\|} B\right\rangle}{I(\psi) p \frac{\partial \ln n}{\partial \psi}}, \\
\left\langle j_{\|} B\right\rangle=-I(\psi) p_{\mathrm{e}}\left(\mathscr{L}_{31}+\mathscr{L}_{32}\right) \frac{\partial \ln T_{\mathrm{e}}}{\partial \psi} \\
\rightarrow \mathscr{L}_{32}=\frac{-\left\langle j_{\|} B\right\rangle}{I(\psi) p_{\mathrm{e}} \frac{\partial \ln T_{\mathrm{e}}}{\partial \psi}}-\mathscr{L}_{31} \\
\left\langle j_{\|} B\right\rangle=-I(\psi) p_{\mathrm{i}}\left(\mathscr{L}_{31}+\mathscr{L}_{34} \alpha\right) \frac{\partial \ln T_{\mathrm{i}}}{\partial \psi} \\
\rightarrow \alpha=\frac{-\left\langle j_{\|} B\right\rangle}{\mathscr{L}_{31} I(\psi) p_{\mathrm{i}} \frac{\partial \ln T_{\mathrm{i}}}{\partial \psi}}-1
\end{gathered}
$$

where the respective equations are already converted directly to the related bootstrap current coefficients. In case of the
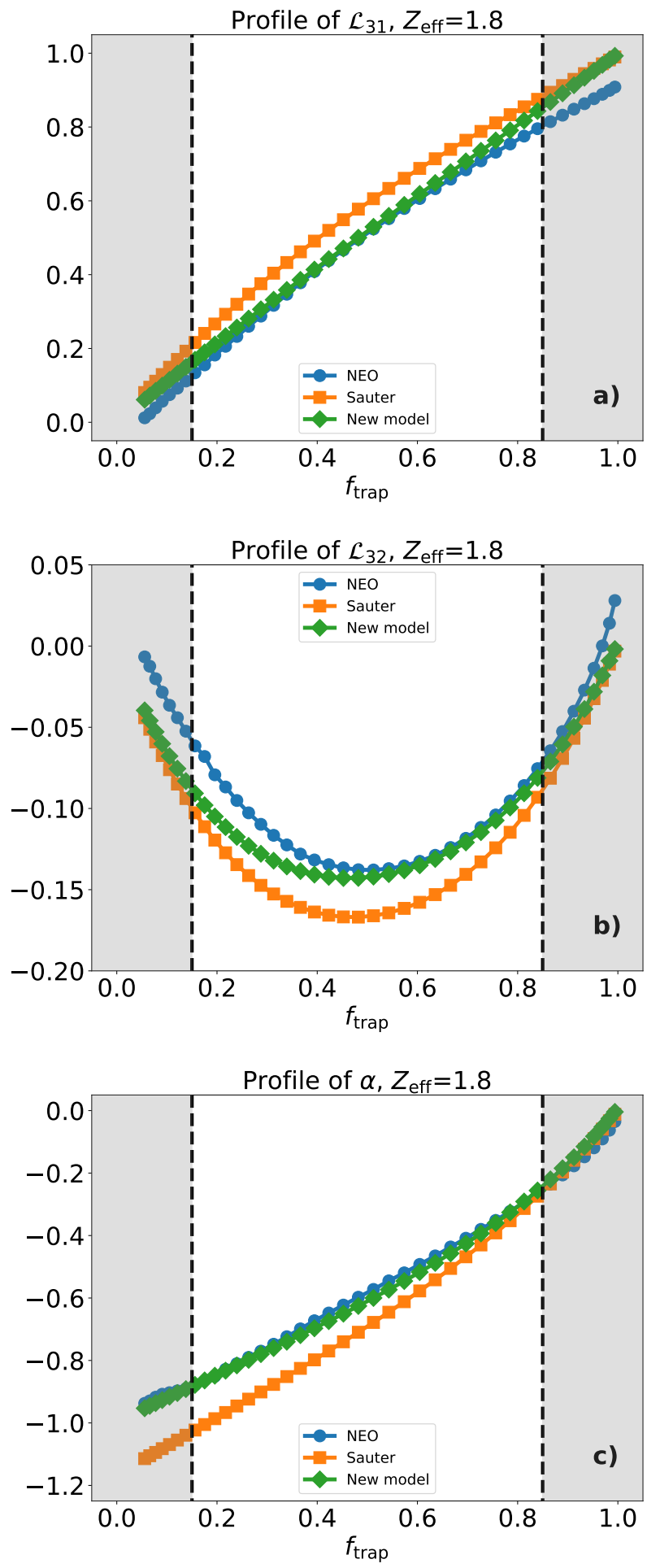

FIG. 3. Profiles of $\mathscr{L}_{31}$ (a), $\mathscr{L}_{32}$ (b) and $\alpha$ (c) in comparison with NEO (solid blue line with circled markers), the original Sauter model (solid orange line with squared markers) and the newly fitted model (solid green line with diamond markers) in the deep banana regime against $f_{\text {trap }}$ for constant $v_{\sigma}^{*}\left(v_{\mathrm{e}}^{*} \approx 8 \cdot 10^{-5}\right.$ and $\left.v_{\mathrm{i}}^{*} \approx 4 \cdot 10^{-5}\right)$, $Z_{\text {eff }}=1.8$ and $\mathrm{N}_{\xi}=39$. It is noted that the grey highlighted areas represents the regions, where the analytic expressions exhibit structural disagreements from the NEO results, which cannot be overcome or avoided. 
Eqs. (4) and (5) the expression of $\mathscr{L}_{31}$ must be substracted. The new model has, in general, the same physics basis as the original Sauter model. However, the code CQLP, which was applied to compute the points used to fit the Sauter model over the full range in collisionality, encountered limitations from both the numerical and the resolution standpoints (with the computational capabilities available more than 20 years ago) in the high collisionality limit. As a consequence the bootstrap current coefficients of the original Sauter model have, in general, clear tendency to be larger in the high collisionality range than those obtained with more recent and reliable solvers.

An additional, and separate, consideration has to be made for the ion flow coefficient $\alpha$. As presented in Ref.[4,5], it was only possible to obtain numerical results for this coefficient up to an intermediate collisionality regime, but not in the highly collisional regime. The high collisionality analytical limit which was used for the Sauter model to obviate the limitations of the code is obtained by considering only ionion collisions. As demonstrated in Refs.[17], the inclusion of ion-electron collisions changes affects this high collisionality limit. Therefore the new model also takes into account the impact of ion-electron collisions with respect to the previous Sauter model.

NEO computes for every involved ion species $\sigma$ its respective flow coefficient. This additional information has to be taken into account into the definition of $\alpha$, since in the original model, only the main ion contribution is considered.

In this new model, we have adopted the simplification of replacing $\mathscr{L}_{34}$ with $\mathscr{L}_{31}$ for all collisionalities. We have determined $\alpha$ in order to correctly reproduce the NEO results for the product $\mathscr{L}_{34} \alpha$, which is the actual factor in front of the ion temperature gradient in the expression of the bootstrap current. Thereby, the coefficient $\alpha$ can be correctly interpreted as providing the customary ion flow only coefficient at low collisionality and low charge number. The new formula of $\alpha$ is obtained in such a way that its product with the new formula for $\mathscr{L}_{31}$ matches the NEO results of $\mathscr{L}_{34} \alpha$. This approach can be used, since the bootstrap current coefficients $\mathscr{L}_{34}$ and $\mathscr{L}_{31}$ are almost identical. Eq. (10) from Ref.[4], which defines the integral of $\mathscr{L}_{34}$, only differs by an additional factor of $B^{2} /\left\langle B^{2}\right\rangle$ from Eq. (8) in Ref.[4], which defines the integral of $\mathscr{L}_{31}$. At $\log _{10}\left(v_{\mathrm{e}}^{*}\right) \approx 0$ the bootstrap current coefficients deviate by $\approx 6 \%$ at the most (according to the relation $\left.\mathscr{L}_{34 \text {,Sauter }} / \mathscr{L}_{31 \text {,Sauter }}-1\right)$. Furthermore, this approach allows one to reduce the number of required analytic formulae.

To determine the neoclassical conductivity $\sigma_{\text {neo }}$ the normalized parallel inductive electric field $\left\langle E_{\|} B\right\rangle$ is set to 1 and the remaining gradients to 0, simplifiying Eq. (2) to the following relation:

$$
\sigma_{\text {neo }}=\left\langle j_{\|} B\right\rangle
$$

With this approach, the sought $\sigma_{\text {neo }}$ can be treated similarly to the $j_{\mathrm{BS}}$-results.

However, this approach has a small disadvantage: $\mathscr{L}_{32}$ can only be broken down and fitted as the sum of the individual contributions $F_{32 \text {,ei }}$ and $F_{32 \text {,ee }}$ (according to Eq. (15a) in Ref.[4]), and not the individual contributions $F_{32 \text {,ei }}$ and $F_{32 \text {,ee, }}$
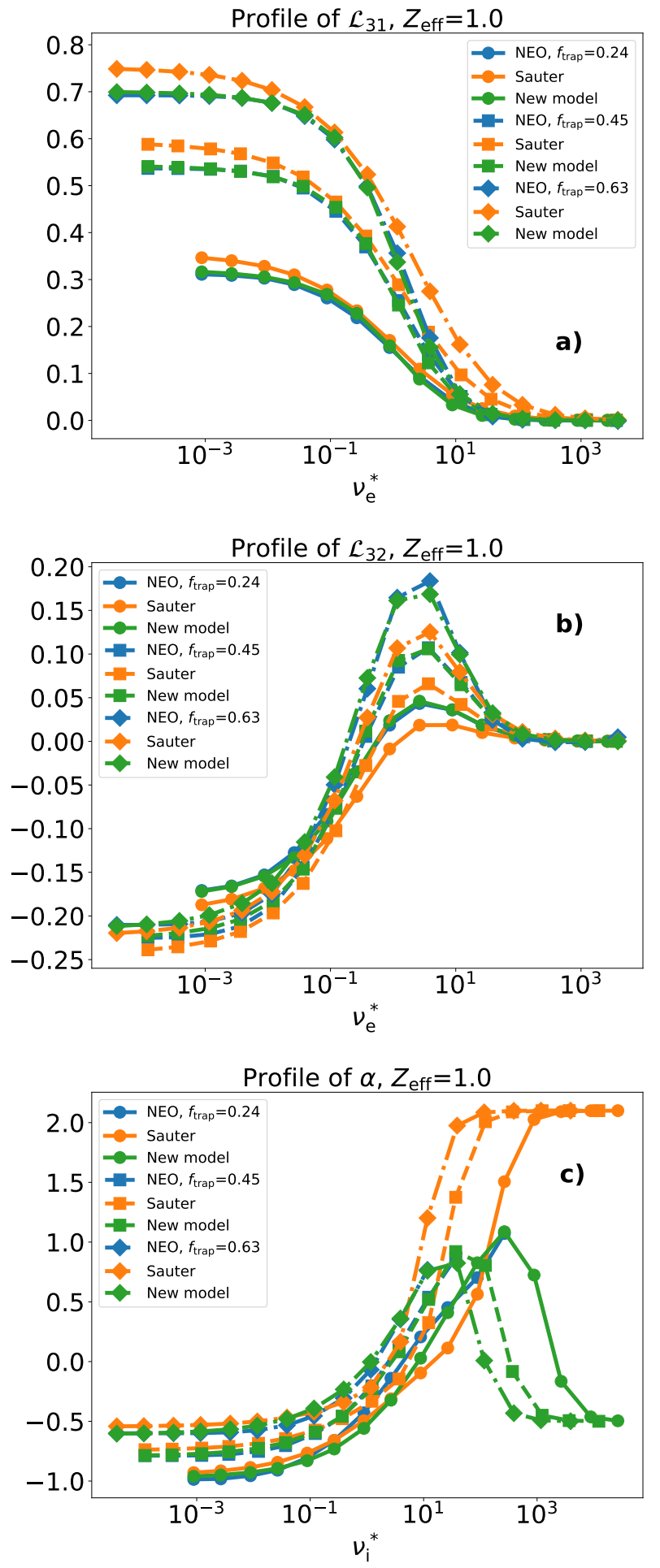

FIG. 4. Profiles of $\mathscr{L}_{31}$ (a), $\mathscr{L}_{32}$ (b) and $\alpha$ (c) in comparison with NEO (blue line), the original Sauter model (orange line) and the newly fitted model (green line) against $v_{\mathrm{e}}^{*}$ for suitably chosen values of $f_{\text {trap }}$, for $Z_{\text {eff }}=1.0$ and $\mathrm{N}_{\xi}=69$. An individual line and marker style is used for the each value of $f_{\text {trap }}$. 
as realized in the original work. With these derived Eqs. (3$6)$, the profiles of the respective bootstrap current coefficients can now be determined from suitably prepared NEO results and exploited as the numerical basis to improve the original analytic formulae.

\section{B. Determination of the bootstrap current coefficients in the banana regime}

In this subsection the determination of the bootstrap current coefficients in the banana regime is discussed. In the next subsection the determination of the bootstrap current coefficients for arbitrary collisionality is discussed. In the last subsection the newly obtained analytic formulae are presented.

To determine the respective bootstrap current coefficients the procedure of Ref.[4] is repeated. Firstly, the analytic expression of the bootstrap current coefficients are fitted in the deep banana regime. In this limit the expressions of the 'effective' trapped particles equations (Eqs. (13b), (14b), (15d), (15e), (16b) and (17b) in Ref.[4]) reduce to the unperturbed (collisionless) expression of $f_{\text {trap }}$. The analytic expression of bootstrap current coefficients (Eqs. (13a), (14a), (15b), (15c) and (17a) in Ref.[4]) can be fitted to the respectively prepared NEO profiles. An acceptable agreement of the respective bootstrap coefficient is achieved, when the new analytic formula matches the numerical results for each coefficient within an error range of $\pm 5 \%$. This modification is obtained in the important ranges of the numerical results and where the analytical structures allow the required agreement. The $Z_{\text {eff-independent prefactors of the polynomial }}$ terms are adjusted in the deep banana regime. An agreement is achieved by modifying the numerical values in the related numerators and denominators. The modified coefficients still fulfill the same analytical limits as the original formulae at $f_{\text {trap }}=0$ and $f_{\text {trap }}=1$. Subsequently, the numerical values related to the $Z_{\text {eff }}$ in the denominators and numerators are modified for a selection of $Z_{\text {eff }}=[1.0,1.4,1.8,2.5,3.5]$. In some cases, the analytical terms containing the parameter $Z_{\text {eff }}$ have been modified. Since the analytic expression of Eq. (17a) from Ref.[4] does not include the effects of multi-species, the analytic epxression of $\alpha_{0}$ is modified by introducing the key input parameter $Z_{\text {eff }}$ into the original analytic expressions.

To fit the analytic formulae, NEO input files are computed with varying $f_{\text {trap }}$, with constant $v_{\sigma}^{*} \ll 0.01$ and circular shaped plasma realised with a Miller-type equilibrium for the selection of $Z_{\text {eff }}$. To calculate cases with $Z_{\text {eff }}>1.0$, a second ion species is implemented. In the presented cases carbon is used as impurity. The inclusion of carbon represents the impact of a low $\mathrm{Z}$ impurity, like typically $\mathrm{B}, \mathrm{C}$ or $\mathrm{N}$ in the experiments. The impact of different mixes of light impurities is just captured by the dependence on $Z_{\text {eff }}$ and main ion, neglecting the slightly different collisionalities of each light impurity. In this way, the input and the output of the new model are the same as in the original Sauter model. In contrast, contributions from high- $Z$ impurities have not been specifically calculated in this work, as these are usually present in trace concentrations only in the experiments, with small to negli-
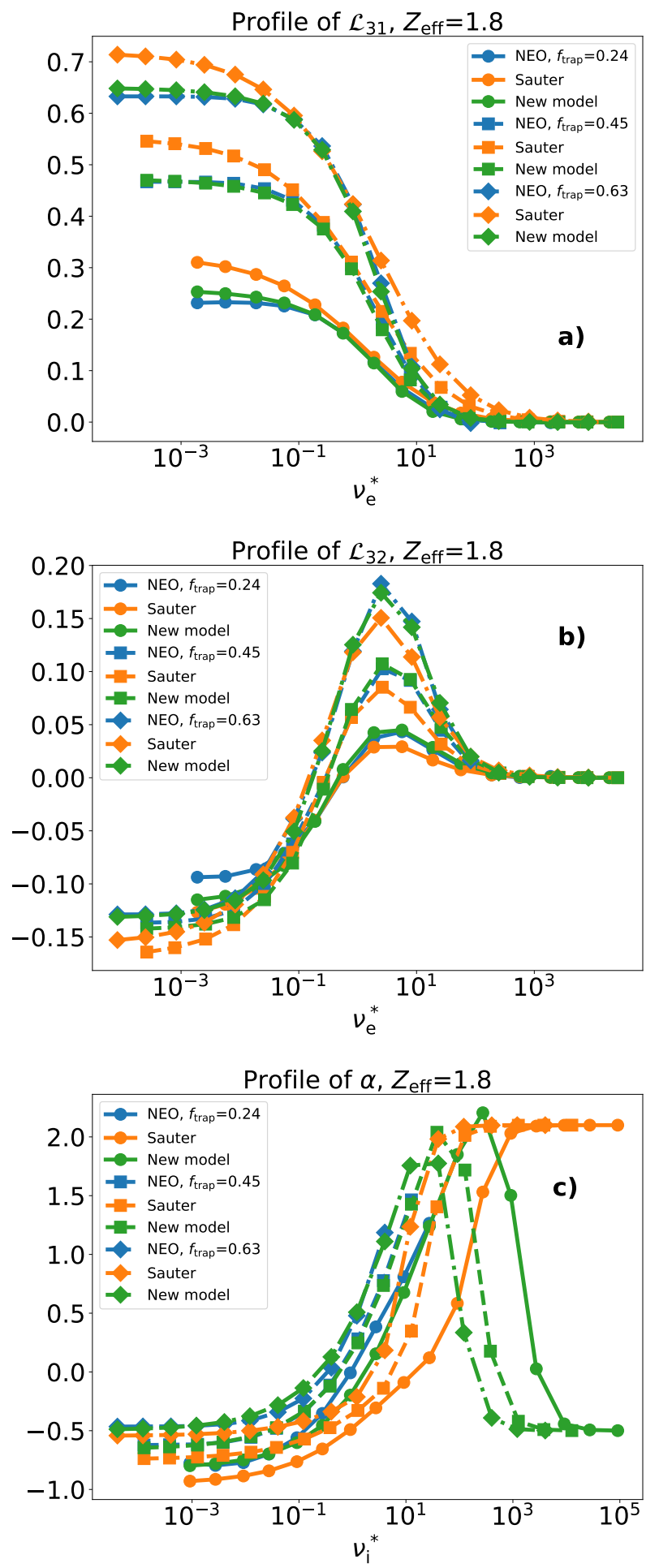

FIG. 5. Profiles of $\mathscr{L}_{31}$ (a), $\mathscr{L}_{32}$ (b) and $\alpha$ (c) in comparison with NEO (blue line), the original Sauter model (orange line) and the newly fitted model (green line) against $v_{\mathrm{e}}^{*}$ for suitably chosen values of $f_{\text {trap }}$, for $Z_{\text {eff }}=1.8$ and $\mathrm{N}_{\xi}=39$. An individual line and marker style is used for the each value of $f_{\text {trap }}$. 
gible impact on the value of $Z_{\text {eff. }}$ In the analytical model, impurities are treated like ions. Therefore, the individual ion contributions (for density, temperature, pressure, gradients in density and temperature) in the respective equations must be replaced by a sum $\sum_{\text {ions, impurities }}$ of the involved ion species:

$$
\begin{gathered}
p_{\mathrm{e}}+p_{\mathrm{i}} \rightarrow p_{\mathrm{e}}+\sum_{\substack{\text { ions, } \\
\text { impurities }}} p_{\sigma} \\
\left(\mathscr{L}_{31}+\mathscr{L}_{34} \alpha\right) p_{\mathrm{i}} \frac{\partial \ln T_{\mathrm{i}}}{\partial \psi} \rightarrow\left(\mathscr{L}_{31}+\mathscr{L}_{34} \alpha\right)\left(\sum_{\text {ions }} p_{\sigma}\right) \frac{\partial \ln T_{\mathrm{i}}}{\partial \psi} .
\end{gathered}
$$

Only the cases $Z_{\text {eff }}=1.0$ and $Z_{\text {eff }}=1.8$ are presented. In addition, only the analytic formulae of $\mathscr{L}_{31}, \mathscr{L}_{32}$ and $\alpha$ are shown. In Fig. 2, the profiles of the numerical solver NEO (solid blue line with squared markers), the original Sauter model (solid orange line with circled markers) and the new model (solid green line with diamond markers) are arranged for comparing these bootstrap coefficients in the deep banana regime for $Z_{\text {eff }}=1.0$. This colour code is kept for now on. The newly obtained analytic formulae are well aligned to the NEO profiles in the relevant range in $f_{\text {trap }}$.

It is noted that the grey highlighted areas represents the regions, where the analytic expressions exhibit structural disagreements from the NEO results, which cannot be overcome or avoided. Thereby, the new formulae cannot follow the numerical values over the full range, since in our approach the new formulae must still follow the analytical limits of the original model. Therefore, close to the boundaries of the value range in $f_{\text {trap }}$ the new model matches the old analytical profiles better than those from NEO. The first grey area contains the values of $f_{\text {trap }} \lesssim 0.15$. This value of $f_{\text {trap }}$ denotes an $\mathrm{r} / \mathrm{a} \approx 0.035$, which is very close to the magnetic axis. The second gray area covers the values for $f_{\text {trap }} \gtrsim 0.85$. Exploiting the large aspect ratio limit, one can use a simple expression to determine the maximum $f_{\text {trap }}$ in conventional tokamaks: $f_{\text {trap,max }} \approx 0.75[20]$. Within these two limits the important regions of conventional tokamaks are covered. Both limit are not fixed, but only a rough definition to be able to divide the regions. These mentioned disagreements usually do not affect the final results, since neither the core (located at low values of $f_{\text {trap }}$ ) nor the plasma edge (located at high values of $f_{\text {trap }}$ ) are located in the deep banana regime. Fig. 3 shows the final analytic formulae for $Z_{\text {eff }}=1.8$. The newly obtained analytic formulae are also well aligned to the NEO profiles in the relevant range in $f_{\text {trap }}$. From Fig. 3 (c), it can be seen that the usage of the parameter $Z_{\text {eff }}$ is a useful parameter to consider multi-species effects in the ion flow coefficient $\alpha$.

\section{Determination of the bootstrap current coefficients for arbitrary $v_{\sigma}^{*}$}

With the newly obtained analytic formulae for the deep banana regime the 'effective' trapped particles equations (Eqs. (13b), (14b), (15d), (15e), (16b) and (17b) from Ref.[4]) for arbitrary collisionality can now be fitted. The 'effective' fraction of trapped particles are fitted over a large range in collisionality $v_{\sigma}^{*}$ and selected values of $f_{\text {trap }}$. The agreements between the analytic formulae and the numerical results for $Z_{\text {eff }}=1.0$ are obtained by modifying the numerical values of the related key parameters $f_{\text {trap }}$ and $v_{\mathrm{e}}^{*}$. The analytical terms containing the key parameters $f_{\text {trap }}$ and $v_{\mathrm{e}}^{*}$ considered in the original 'effective' trapped particles equations are modified, if necessary. If needed, additional analytical terms containing these two key parameters are introduced in the formulae. Afterwards, the 'effective' fraction of trapped particles are fitted . for the selection of $Z_{\mathrm{eff}}$. In some cases, the analytical terms containing the key parameter $Z_{\text {eff }}$ have been modified.

To fit the analytic formulae, NEO input files are computed with varying $v_{\sigma}^{*}$, with a set of values of $f_{\text {trap }}=[0.24,0.45$, 0.63 ] and circular shaped plasma realised with a Miller-type equilibrium for the selection of $Z_{\text {eff. }}$. The other required pa-

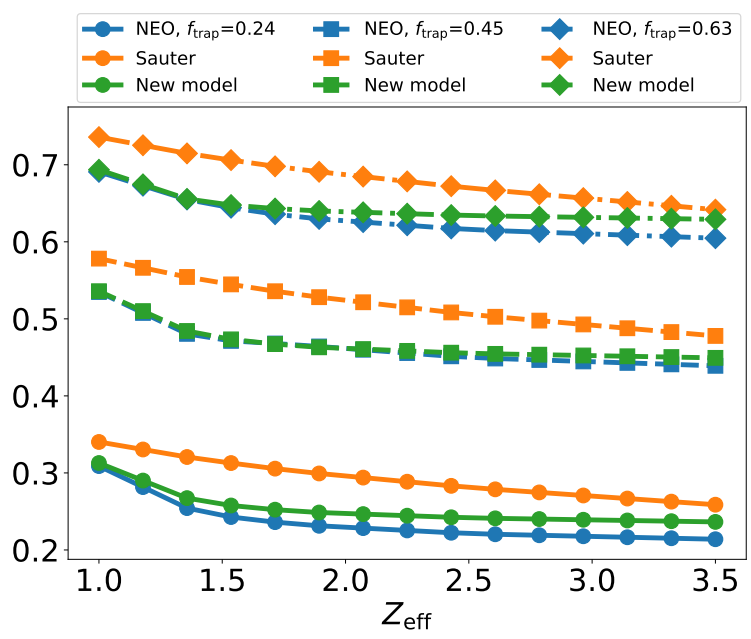

FIG. 6. Profiles of $\mathscr{L}_{31}$ in comparison with NEO (solid blue line with circled markers), the original Sauter model (solid orange line with squared markers) and the newly fitted model (solid green line with diamond markers) against $Z_{\text {eff }}$ for suitably chosen values of $f_{\text {trap }}$. The chosen value of $v_{\mathrm{e}}^{*}$ is located in the banana regime.

rameters are identical to those from the preparations of the previous subchapter. Only the cases $Z_{\text {eff }}=1.0$ and $Z_{\text {eff }}=1.8$ are presented. In addition, only the newly obtained analytic formulae of $\mathscr{L}_{31}, \mathscr{L}_{32}$ and $\alpha$ are presented as previously. In Fig. 4, the profiles of the numerical solver NEO, the original Sauter model and the new model are presented for comparing these discussed bootstrap current coefficients for arbitrary $v_{\sigma}^{*}$ and for $Z_{\text {eff }}=1.0$. Fig. 5 shows the respective profiles for $Z_{\text {eff }}=1.8$. The used colour code is the same as for the previous figures. For different values of $f_{\text {trap }}$ different line styles and markers are used: for $f_{\text {trap }}=0.24$ a solid line with circled markers is used, for $f_{\text {trap }}=0.45$ a dashed line with squared markers is used and for $f_{\text {trap }}=0.63$ a dash-dotted line with diamond markers is used. The newly obtained analytic formulae are well aligned to the NEO profiles over the total range in $v_{\sigma}^{*}$. In case of the ion flow coefficient $\alpha$ a new limit has been introduced. The original limit, which approaches 2.1 , is obtained for a reduced collision operator, which neglects ion-electron 
coupling. The consequences of including ion-electron coupling on the overall behaviour of $\alpha$ has already been discussed and explained in detail in Ref.[14]. The result from Fig. 8 (top left) of Ref.[14] is used as the new limit value, which reaches the value -0.5 . Numerical results at very high collisionality, which were inconsistent with this limit and affected from an inadequate resolution, have been neglected in obtaining the final formulae. Figs. 4 a) and 5 a) easily visualize the reason for the structural overestimation of the bootstrap current by the analytical Sauter model at high collisionality. The inaccuracy in the original numerical profiles can be attributed to the difficulty of reliably running the code CQLP at high collisionality.

The behaviour of the bootstrap current coefficient $\mathscr{L}_{31}$ in the low collisionality limit for a selection of $Z_{\text {eff }}$ is briefly discussed. The related plot for chosen values of $f_{\text {trap }}$ and a chosen value of $v_{\mathrm{e}}^{*}$ in the banana limit against $Z_{\text {eff }}$ can be seen in Fig. 6. The same color code as in the previous plots is used. One can see that in the region of low values of $Z_{\text {eff }}$ the revised bootstrap current transport coefficient $\mathscr{L}_{31}$ is well aligned to the numerically determined results. With increasing $Z_{\text {eff }}$, the agreement between the revised bootstrap current transport coefficient $\mathscr{L}_{31}$ and numerically determined one decreases. The revised coefficient tends more and more to the profiles of the original formula of $\mathscr{L}_{31}$. This problem can be led back to the analytical expression of $\mathscr{L}_{31}$ (Eq. (10)). The $Z_{\text {eff-dependence }}$ of the analytic expression of bootstrap current coefficient $\mathscr{L}_{31}$ is located in the denominator, in the limit $Z_{\text {eff }} \rightarrow \infty$ one obtains the subsequent expression:

$$
\lim _{Z_{\text {eff }} \rightarrow \infty} \mathscr{L}_{31} \rightarrow X_{31}\left(=f_{\text {trap }}\right),
$$

The increasing $Z_{\text {eff }}$ reduces the analytic formula of the bootstrap current coefficient $\mathscr{L}_{31}$ to a remaining $Z_{\text {eff-indepedent }}$ linear contribution, which yields in the limit of low collisionality just $f_{\text {trap }}$. Note that this is because it recovers the Lorentz model (Eq. (15c) of Ref[21,22]. Consequently, these structural disagreements cannot be overcome or avoided. A similar behaviour would be seen for Eq. (17), since the formula has a similar analytic dependence on $Z_{\text {eff }}$ as $\mathscr{L}_{31}$.

It should be noted that for a precise calculation of the bootstrap current exact analytic formulae are required, even in the high collisionality range $\left(v_{\mathrm{e}}^{*}>5\right)$, since the highly interesting plasma edge is an area of high collisionality. The aligned contributions of the most important bootstrap current coefficient $\mathscr{L}_{31}$ are very small in this high collisionality range. But due to steep density and temperature gradients $\left(\mathrm{d} \ln \mathrm{n}_{\sigma} / \mathrm{d} \psi\right.$ and $\mathrm{d} \ln \mathrm{T}_{\sigma} / \mathrm{d} \psi>10$ ) occuring at the plasma edge, significant and non-negligible current contributions can still be generated.

\section{Newly obtained analytic formulae of the bootstrap current coefficients}

The following formulae are the results of the modifications of the analytical Sauter formulae used so far. It should be noted that the definitions of the key parameters fraction of trapped particles $f_{\text {trap }}$, the collisionality $v_{\sigma}^{*}$ and the effective charge number $Z_{\text {eff }}$ (Eqs. (12), (18a)-(18e) in Ref.[4]) must be used in order to calculate correctly and accurately the bootstrap current density with this set of analytical formulae. The analytical fits to the results of the numerical solver NEO, valid for arbitrary $f_{\text {trap }}, v_{\sigma}^{*}$, and $Z_{\text {eff }}$ are given as follows:

$$
\begin{aligned}
& \mathscr{L}_{31}\left(X_{31}=f_{\mathrm{t}, 31}^{\mathrm{eff}}\right)=\left(1+\frac{0.15}{Z_{\mathrm{eff}}^{1.2}-0.71}\right) X_{31}-\frac{0.22}{Z_{\text {eff }}^{1.2}-0.71} X_{31}{ }^{2}+\frac{0.01}{Z_{\mathrm{eff}}^{1.2}-0.71} X_{31}{ }^{3}+\frac{0.06}{Z_{\mathrm{eff}}^{1.2}-0.71} X_{31}{ }^{4} \\
& f_{\mathrm{t}, 31}^{\mathrm{eff}}=\frac{f_{\text {trap }}}{1+\frac{0.67\left(1-0.7 f_{\text {trap }}\right) \sqrt{v_{\mathrm{e}}^{*}}}{0.56+0.44 Z_{\mathrm{eff}}}+\frac{\left(0.52+0.086 \sqrt{v_{\mathrm{e}}^{*}}\right)\left(1+0.87 f_{\text {trap }}\right) v_{\mathrm{e}}^{*}}{1+1.13\left(Z_{\mathrm{eff}}-1\right)^{0.5}}} \\
& \mathscr{L}_{32}=F_{32, \mathrm{ei}}+F_{32, \mathrm{ee}} \\
& F_{32, \mathrm{ee}}\left(X_{32, \mathrm{e}}=f_{\mathrm{t}, 32, \mathrm{ee}}^{\mathrm{eff}}\right)=\frac{0.1+0.6 Z_{\mathrm{eff}}}{Z_{\mathrm{eff}}\left(0.77+0.63\left(1+\left(Z_{\mathrm{eff}}-1\right)^{1.1}\right)\right)}\left(X_{32, \mathrm{e}}-X_{32, \mathrm{e}}{ }^{4}\right)+ \\
& \frac{0.7}{1+0.2 Z_{\text {eff }}}\left(X_{32, \mathrm{e}^{2}}-X_{32, \mathrm{e}^{4}}-1.2\left(X_{32, \mathrm{e}^{3}}-X_{32, \mathrm{e}^{4}}\right)\right)+\frac{1.3}{1+0.5 Z_{\mathrm{eff}}} X_{32, \mathrm{e}}{ }^{4} \\
& f_{\mathrm{t}, 32, \mathrm{ee}}^{\mathrm{eff}}=\frac{f_{\text {trap }}}{1+\frac{0.23\left(1-0.96 f_{\text {trap }}\right) \sqrt{v_{\mathrm{e}}^{*}}}{Z_{\mathrm{eff}}^{0.5}}+\frac{0.13\left(1-0.38 f_{\text {trap }}\right) v_{\mathrm{e}}^{*}}{Z_{\mathrm{eff}}^{2}}\left(\sqrt{1+2\left(Z_{\mathrm{eff}}-1\right)^{0.5}}+f_{\text {trap }}^{2} \sqrt{\left(0.075+0.25\left(Z_{\mathrm{eff}}-1\right)^{2}\right) v_{\mathrm{e}}^{*}}\right)} \\
& F_{32, \mathrm{ei}}\left(X_{32, \mathrm{ei}}=f_{\mathrm{t}, 32, \mathrm{ei}}^{\mathrm{eff}}\right)=-\frac{0.4+1.93 Z_{\mathrm{eff}}}{Z_{\mathrm{eff}}\left(0.8+0.6 Z_{\mathrm{eff}}\right)}\left(X_{32, \mathrm{ei}}-X_{32, \mathrm{ei}}{ }^{4}\right)+ \\
& \frac{5.5}{1.5+2 Z_{\mathrm{eff}}}\left(X_{32, \mathrm{ei}}{ }^{2}-X_{32, \mathrm{ei}}{ }^{4}-0.8\left(X_{32, \mathrm{ei}^{3}}{ }^{3} X_{32, \mathrm{ei}}{ }^{4}\right)\right)-\frac{1.3}{1+0.5 Z_{\mathrm{eff}}} X_{32, \mathrm{ei}}{ }^{4}
\end{aligned}
$$




$$
\begin{gathered}
f_{\mathrm{t}, 32, \mathrm{ei}}^{\mathrm{eff}}=\frac{f_{\text {trap }}}{1+\frac{0.87\left(1+0.39 f_{\text {trap }}\right) \sqrt{v_{\mathrm{e}}^{*}}}{1+2.95\left(Z_{\mathrm{eff}}-1\right)^{2}}+1.53\left(1-0.37 f_{\text {trap }}\right) v_{\mathrm{e}}^{*}\left(2+0.375\left(Z_{\mathrm{eff}}-1\right)\right)} \\
\frac{\sigma_{\text {neo }}}{\sigma_{\text {Spitzer }}}\left(X_{33}=f_{\mathrm{t}, 33}^{\mathrm{eff}}\right)=1-\left(1+\frac{0.21}{Z_{\mathrm{eff}}}\right) X_{33}+\frac{0.54}{Z_{\mathrm{eff}}} X_{33}{ }^{2}-\frac{0.33}{Z_{\mathrm{eff}}} X_{33^{3}} \\
f_{\mathrm{t}, 33}^{\mathrm{eff}}=\frac{f_{\text {trap }}}{1+0.25\left(1-0.7 f_{\text {trap }}\right) \sqrt{v_{\mathrm{e}}^{*}}\left(1+0.45\left(Z_{\mathrm{eff}}-1\right)^{0.5}\right)+\frac{0.61\left(1-0.41 f_{\text {trap }}\right) v_{\mathrm{e}}^{*}}{Z_{\text {eff }}{ }^{*}}} \\
\alpha_{0}=-\frac{0.62+0.055\left(Z_{\mathrm{eff}}-1\right)}{0.53+0.17\left(Z_{\mathrm{eff}}-1\right)} \frac{1-f_{\text {trap }}}{1-\left(0.31-0.065\left(Z_{\text {eff }}-1\right)\right) f_{\text {trap }}-0.25 f_{\text {trap }}{ }^{2}} \\
\alpha=\left(\frac{\alpha_{0}+0.7 Z_{\text {eff }} f_{\text {trap }} 0.5 \sqrt{v_{\mathrm{i}}^{*}}}{1+0.18 \sqrt{v_{\mathrm{i}}^{*}}}-0.002 v_{\mathrm{i}}^{* 2} f_{\text {trap }}{ }^{6}\right) \frac{1}{1+0.004 v_{\mathrm{i}}^{* 2} f_{\text {trap }}{ }^{6}}
\end{gathered}
$$

\section{APPLICATION OF THE MODELS TO A TYPICAL ASDEX UPGRADE DISCHARGE}

In order to present the improvements of the revised model a typical discharge of ASDEX Upgrade is considered. ASDEX Upgrade discharge \#33173 has been presented in a previous paper[23] to analyze the crucial role of the pedestal pressure on the plasma edge stability in ASDEX Upgrade. It is an H-mode plasma with $I_{\mathrm{P}}=1 \mathrm{MA}, B_{\text {tor }}=|2.5| \mathrm{T}$ and $P_{\text {Heat }}=12 \mathrm{MW}$. In addition, rates of the gas puff are varied. Fig. 2 in Ref.[23] summarizes the time traces of the most important parameters. A time point at $4.75 \mathrm{~s}$ is chosen, where a medium gas fuelling rate is applied. Fig. 7 (a) presents profiles of the numerical solver NEO, the original Sauter model and the revised model. Since the deviations, particularly for $\rho_{\text {pol }}<0.95$, are very small, a second plot (Fig. 7 (b)), where the relation $\left(j_{\mathrm{BS} \text {,model }} / j_{\mathrm{BS}, \mathrm{NEO}}-1\right)$ is exploited, is used in order to visualize the respective differences of the original Sauter model and the revised model from the NEO results. The Fig. 7 (c) presents the profiles of $v_{\mathrm{e}}^{*}, v_{\mathrm{i}}^{*}$, $-E_{\text {radial }} /\left(v_{\text {th,i }} \cdot B_{\text {pol }}\right), \mathrm{R} / L_{\mathrm{n}_{\mathrm{e}}}, \mathrm{R} / L_{\mathrm{T}_{\mathrm{i}}}, \rho_{\theta, \mathrm{i}} / L_{\mathrm{n}_{\mathrm{e}}}$ and $\rho_{\theta, \mathrm{i}} / L_{\mathrm{T}_{\mathrm{i}}}$ for the ASDEX Upgrade discharge to gauge the potential significance of non-local effects at the plasma edge. Here $\rho_{\theta, \mathrm{i}}$ is the poloidal Larmor radius, $1 / L_{T_{i}}=-\mathrm{d} \ln \left(\mathrm{T}_{\mathrm{i}}\right) / \mathrm{d} r$ and $1 / L_{n_{e}}=$ $-\mathrm{d} \ln \left(\mathrm{n}_{\mathrm{e}}\right) / \mathrm{d} r$. The code NEO is a local code and its solution for the ion distribution might be not valid at the plasma edge, since the perturbative formalism with the ordering parameter $\rho_{\mathrm{i}}^{*}$ could break down in this region[17]. As seen in Fig. 8 (Top profile) the current contribution connected with the ion distribution function delivers the smallest contribution to the total current. But any corrections of the ion distribution through the consideration of non-local effects might affect the total results. Nevertheless, one can see that for $\rho_{\text {pol }}<0.95$ the re- vised model has a reliable agreement with the results of NEO and the original Sauter model. The disagreement is $\ll 5 \%$. For $\rho_{\text {pol }}>0.95$, one sees a significant agreement of the revised model with the NEO results. Except for the last value $(\approx 0.995$ and $30 \%$ disagreement) all remaining points agree within an error range of $10 \%$ from the NEO results, whereas the original Sauter model disagrees up to $100 \%$ from the numerical results. Two possible arrangements of the current contributions are used to visualize the improvements of the newly fitted model. The Fig. 8 (top) presents the bootstrap current contributions associated to the bootstrap current coefficients $\mathscr{L}_{31}, \mathscr{L}_{32}, \mathscr{L}_{34} \alpha$ (see Eq. (2)). The current contributions are calculated with the code NEO (dotted line), the original Sauter model (dashed line) and the revised model (solid line). The blue line denotes the bootstrap current contribution of the bootstrap current coefficient $\mathscr{L}_{31}$, the orange line the one of the coefficient $\mathscr{L}_{32}$ and the green line the one of the coefficient $\mathscr{L}_{34} \alpha$. For the current contribution connected with the coefficient $\mathscr{L}_{31}$, one can see a strongly lowered current density calculated with the revised model for $\rho_{\text {pol }}>0.9$. The current contribution connected with the coefficient $\mathscr{L}_{32}$ calculated with both models matches each other almost perfectly. A slight difference between these two profiles can only be observed around $\rho_{\text {pol }} \approx 0.97$. Interesting changes can be seen in the current density profiles of the coefficient $\mathscr{L}_{34} \alpha$, where unexptected large disagreements between the analytical models can be observed for $\rho_{\text {pol }}>0.9$, which can be led back to the consideration of impurities in the revised formula of $\alpha$. In Fig. 8 (bottom), the respective current contributions of the bootstrap current are arranged with the respect to the driving gradients. The blue line denotes the contribution associated with the electron density gradient, the orange one with the ion density gradient, the green one with the electron tem- 

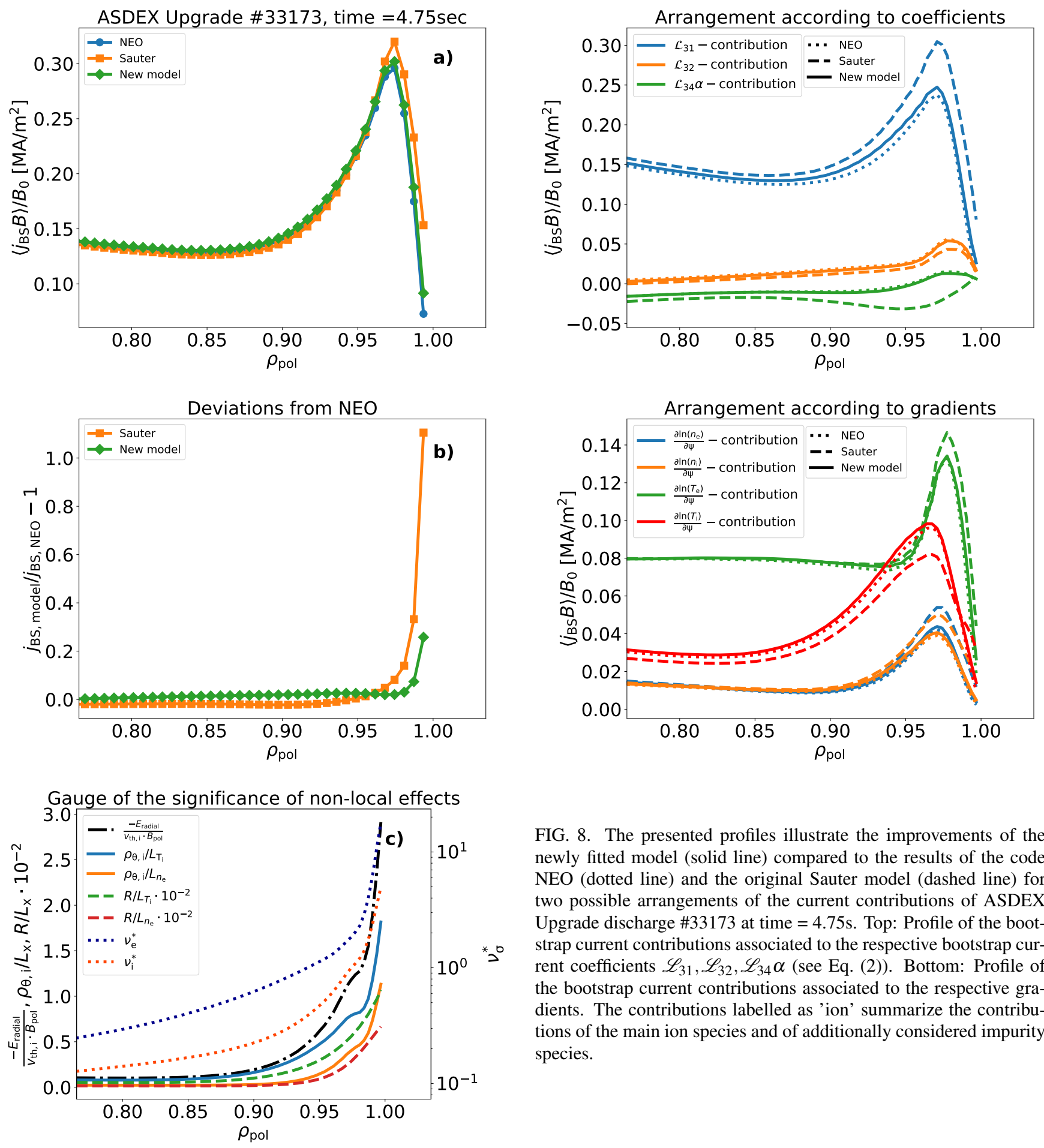

FIG. 8. The presented profiles illustrate the improvements of the newly fitted model (solid line) compared to the results of the code NEO (dotted line) and the original Sauter model (dashed line) for two possible arrangements of the current contributions of ASDEX Upgrade discharge \#33173 at time $=4.75$ s. Top: Profile of the bootstrap current contributions associated to the respective bootstrap current coefficients $\mathscr{L}_{31}, \mathscr{L}_{32}, \mathscr{L}_{34} \alpha$ (see Eq. (2)). Bottom: Profile of the bootstrap current contributions associated to the respective gradients. The contributions labelled as 'ion' summarize the contributions of the main ion species and of additionally considered impurity species.

FIG. 7. Plot a): Profile of ASDEX Upgrade discharge \#33173 at time $=4.75 \mathrm{~s}$ comparing NEO (solid blue line with circled markers), the original Sauter model (solid orange line with squared markers) and the newly fitted model (solid green line with diamond markers) against $0.75<\rho_{\text {pol }}<1.00$. Plot b): Profile of the same discharge comparing the differences of the original Sauter model (solid orange line with squared markers) and the newly fitted model (solid green line with diamond markers) from the NEO results against $0.75<$ $\rho_{\text {pol }}<1.00$. Plot c): Profiles of $v_{\mathrm{e}}^{*}, v_{\mathrm{i}}^{*},-E_{\text {radial }} /\left(v_{\text {th,i }} \cdot B_{\text {pol }}\right), \mathrm{R} / L_{\mathrm{n}_{\mathrm{e}}}$, $\mathrm{R} / L_{\mathrm{T}_{\mathrm{i}}}, \rho_{\theta, \mathrm{i}} / L_{\mathrm{n}_{\mathrm{e}}}$ and $\rho_{\theta, \mathrm{i}} / L_{\mathrm{T}_{\mathrm{i}}}$ for the ASDEX Upgrade discharge to gauge the potential significance of non-local effects at the plasma edge.

perature gradient and the red one with the ion temperature gradient. One can see an unexpected and significant increase in the current contribution generated by the ion temperature gradient. It must be mentioned, that the significance of this term might make non-local effects important[13], which cannot be considered in this study. The three remaining profiles show slightly lowered maximum peaks in the respective profile around $\rho_{\text {pol }} \approx 0.97$. These promising improvements could lead to interesting consequences on the stability analysis of plasmas in ASDEX Upgrade. 


\section{SUMMARY AND OUTLOOK}

A revised model for calculating the bootstrap current and the neoclassical conductivity has been derived and presented following the analogous methodology (exploiting the same analytical structure and key parameters $\left.\left(f_{\text {trap }}, v_{\sigma}^{*}, Z_{\text {eff }}\right)\right)$ of Ref.[4], but applied to the numerical results of NEO. Starting from Eq. (2), the respective bootstrap current coefficients (Eqs. (3-5)) have been determined from prepared NEO results and used to fit the analytic formulae within an error range of $\pm 5 \%$. The procedure to determine the bootstrap current coefficients in Ref.[4] is repeated with the more accurate and reliable numerical code NEO in order to determine suitable analytical formulae (Eqs. (13-17)) of Ref.[4], which do not suffer on the structural disagreements discussed in Ref.[14]. For the presented results, the definitions of the mentioned key parameters $f_{\text {trap }}, v_{\sigma}$ and $Z_{\text {eff }}$, as given in Ref.[4], are used. Additionally, exploiting the linearity of neoclassical transport theory is a suitable approach to determine the neoclassical transport coefficients from prepared numerical results. No further fit parameters, without or even with physical sense, must be introduced to fit the analytical formulae of the neoclassical bootstrap current coefficients. According to this, a new set of analytic formulae of the bootstrap current coefficients (Eqs. (10), (13), (15), (17) and (20)) and the related 'effective' trapped particles equations (Eqs. (11), (14), (16), (18) and (21)) have been derived.

Additionally, it would be useful to extend this model to include further physical effects[11, 12, 13], which cannot be considered through the conventional neoclassical orderings used in the code NEO.

It may now become interesting to apply this revised model to further tokamak experiments. Next to this, it is added that the bootstrap current coefficient $\mathscr{L}_{31}$ can also be exploited to determine the neutral beam drivent current efficiency factor $\eta_{\mathrm{NBCD}}[24]$, which in particular is used in the numerical code NUBEAM[25,26] and the real-time simulation code RABBIT[27]. Furthermore, the results of Fig. 7 motivate us to consider the newly obtained set of analytic formulae as a subroutine on the workflow on examining the stability analysis of the pedestal pressure at ASDEX Upgrade discharges[23]. Finally, we recall that the coefficient $\mathscr{L}_{31}$ can be used for the calculation of the Ware pinch, since conventional neoclassical transport theory satisfies the Onsager symmetry $\left(\mathscr{L}_{31}=\mathscr{L}_{13}\right)[21,22]$.

\section{ACKNOWLEDGMENTS}

The authors are grateful to M. Dunne, R. Fischer, M. Weiland and $\mathrm{H}$. Zohm for very fruitful discussions.

This work has been carried out within the framework of the EUROfusion Consortium and has received funding from Euratom research and training programme 2014-2018 and 20192020 under grant agreement No. 633053. The views and opinions expressed herein do not necessarily reflect those of the European Commission. OS was supported in part by the
Swiss National Science Foundation.

\section{DATA AVAILABILITY}

The data that support the findings of
this study are openly available in at
https://doi.org/10.5281/zenodo.4066956.(Ref[28])

\section{REFERENCES}

${ }^{1}$ A. A. Galeev and R. Z. Sagdeev, Soviet Physics - JETP 26, 233 (1968).

${ }^{2}$ R. J. Bickerton, J. W. Connor, and J. B. Taylor, Nature Physical Science 229, 110 (1971).

${ }^{3}$ A. G. Peeters, Plasma Physics and Controlled Fusion 42, B231 (2000).

${ }^{4}$ O. Sauter, C. Angioni, and Y. R. Lin-Liu, Physics of Plasma 6, 2834 (1999).

${ }^{5}$ O. Sauter, C. Angioni, and Y. R. Lin-Liu, Physics of Plasma 9, 5140 (2002).

${ }^{6}$ J. Killeen, G. D. Kerbel, M. G. McCoy, and A. A. Mirin, Computational Methods for Kinetic Models of Magnetically Confined Plasmas, SpringerVerlag (1986).

${ }^{7}$ R. W. Harvey and M. G. McCoy, International Atomic Energy Agency, 489-526 (1992).

${ }^{8}$ O. Sauter, R. W. Harvey, and F. L. Hinton, Contribution to Plasma Physics 34, 169 (1994).

${ }^{9}$ S. P. Hirshman and D. J. Sigmar, Nuclear Fusion 21, 1079 (1981).

${ }^{10}$ F. L. Hinton and R. D. Hazeltine, Reviews of Modern Physics 48, 239 (1976).

${ }^{11}$ S. Koh, C. S. Chang, S. Ku, J. E. Menard, H. Weitzner, and W. Choe, Physics of Plasmas 19, 072505 (2012).

${ }^{12}$ R. Hager and C. S. Chang, Physics of Plasmas 23, 042503 (2016).

${ }^{13}$ M. Landreman and D. R. Ernst, Plasma Physics and Controlled Fusion 54, 115006 (2012).

${ }^{14}$ E. A. Belli, J. Candy, O. Meneghini, and T. H. Osborne, Plasma Physics and Controlled Fusion 56, 045006 (2014).

${ }^{15}$ E. A. Belli and J. Candy, Plasma Physics and Controlled Fusion 50, 095010 (2008).

${ }^{16}$ E. A. Belli and J. Candy, Plasma Physics and Controlled Fusion 51, 075018 (2009).

${ }^{17}$ E. A. Belli and J. Candy, Plasma Physics and Controlled Fusion 54, 015015 (2012).

${ }^{18}$ S. I. Braginskii, Reviews of Plasma Physics 1, 205 (1965).

${ }^{19}$ W. A. Houlberg, K. C. Shaing, S. P. Hirshman, and M. C. Zarnstorff, Physics of Plasmas 4, 3230 (1997).

${ }^{20}$ Y. B. Kim, P. H. Diamond, and R. J. Groebner, Physics of Fluids 3, 2050 (1991).

${ }^{21}$ C. Angioni and O. Sauter, Physics of Plasmas 7, 1224 (2000).

${ }^{22}$ C. Angioni and O. Sauter, Physics of Plasmas 7, 3122 (2000).

${ }^{23}$ M. G. Dunne, S. Potzel, F. Reimold, M. Wischmeier, E. Wolfrum, L. Frassinetti, M. Beurskens, P. Bilkova, M. Cavedon, R. Fischer, B. Kurzan, F. M. Laggner, R. M. McDermott, G. Tardini, E. Trier, E. Viezzer, M. Willensdorfer, the EUROfusion MST1 Team, and the ASDEX-Upgrade Team, Plasma Physics and Controlled Fusion 59, 014017 (2017).

${ }^{24}$ Y. R. Lin-Lui and F. L. Hinton, Physics of Plasmas 4, 4179 (1997).

${ }^{25}$ R. J. Goldston, D. C. McCune, H. H. Towner, S. L. Davis, R. J. Hawryluk, and G. L. Schmidt, Journal of Computational Physics 43, 61 (1981).

${ }^{26}$ A. Pankin, D. McCune, R. Andre, G. Bateman, and A. Kritz, Computer Physics Communications 159, 157 (2004).

${ }^{27}$ M. Weiland, R. Bilato, R. Dux, B. Geiger, A. Lebschy, F. Felici, R. Fischer, D. Rittich, M. van Zeeland, the ASDEX Upgrade Team, and the Eurofusion MST1 Team, Nuclear Fusion 58 (2018).

${ }^{28}$ A. Redl, C. Angioni, E. Belli, O. Sauter, the ASDEX Upgrade Team, and the EUROfusion MST1 Team, "A new set of analytical formulae for the computation of the bootstrap current and the neoclassical conductivity in tokamaks," Zenodo, http://doi.org/10.5281/zenodo.4072358 (2020). 